\title{
Impact of the Skin Disinfection with Quaternary Ammonium Salts on the Rheological Properties of Hyaluronic Acid Viscosupplements
}

\author{
Elise Murat ${ }^{1}$ and Thierry Conrozier ${ }^{1,2 *}$ \\ ${ }^{1}$ Laboratoire d'Exploration et Morphométrie Articulaire (LEMA), France
}

${ }^{2}$ Service de rhumatologie, Hôpital Nord Franche-Comté, France

*Corresponding author: Thierry Conrozier, Laboratoire d'Exploration et Morphométrie Articulaire (LEMA), France; Service de rhumatologie, Hôpital Nord Franche-Comté, France

\section{ARTICLE INFO}

Received: April 12, 2021

Published: 蔧 May 06, 2021

Citation: Elise Murat, Thierry Conrozier Impact of the Skin Disinfection with Quaternary Ammonium Salts on the Rheological Properties of Hyaluronic Acid Viscosupplements. Biomed J Sci \& Tech Res 35(4)-2021. BJSTR. MS.ID.005728.

Abbreviations: HA: Hyaluronic Acid; QAS: Quaternary Ammonium Salts; OA: Osteoarthritis; IA: Intra-Articular

\begin{abstract}
Background: There is a known incompatibility between hyaluronic acid (HA) and quaternary ammonium salts (QAS) due to the risk of HA precipitation in the solution. Therefore, many instructions for use of HA viscosupplements recommend to avoid the use of QAS to disinfect the injection site. However, in the conditions of clinical practice, it has never been evidenced that QAS disinfectants exert a negative impact on the rheological properties of viscosupplements.
\end{abstract}

Objective: To compare the rheological properties of a cross-linked and a linear HA viscosupplements, before and after different skin disinfection protocols.

Methods: The rheological properties of a cross-linked (HANOX-M) and a linear HA (HANOX-M-XL) were measured using a cone-plate rheometer at $20{ }^{\circ} \mathrm{C}$ after injection through a piece of pork skin previously disinfected with a QAS and an iodinated derivative disinfectant. The same measures after injection, directly on the rheometer plate and through a pork skin not disinfected, were used as controls. The dynamic viscosity $\eta$ (Pa.s) was determined according to the shear rate $\gamma$ from 0.1 to $100 \mathrm{~s}^{-1}$.

Results: The steady-state viscosity as a function of the shear rate of HANOX-M$\mathrm{XL}$ and HANOX-M were not significantly modified regardless of the skin disinfection protocol. The mean $\eta$ (Pa.s) at $\gamma=0.5 \mathrm{~s}^{-1}$ (SD) of the crosslinked HA was respectively 116.2 (5.9), 109.8 (2.8) and 114.4 (4.9) for no disinfection, QAS and iodinated derivative disinfectants $(\mathrm{p}=0.20)$. For linear HA it was respectively 39.2 (1.7), 35.4 (1.8) and 33.8 (0.1) $(\mathrm{p}=0.4)$.

Conclusion: Skin disinfection with QAS does not modify the rheological properties of HA viscosuppléments. The use of QAS should no longer be contraindicated for skin disinfection before HA intra-articular injection.

\section{Introduction}

Viscosupplementation with intra-articular injection(s) of high molecular weight hyaluronic acid (HA) is a widely used and effective therapy for knee osteoarthritis (OA) [1]. Hyaluronic acid products are constituted of a solution of linear or crosslinked HA and are administered intra-articularly using a rigorous protocol of asepsis [2], the only potentially serious adverse event being joint infection, which is rare and directly dependent upon the number of injections [3]. Regardless the treatment injected, the skin has to be carefully disinfected before intra-articular (IA) injection for preventing the passage of skin bacteria into the joint cavity, which is the main severe adverse event of IA therapies [4]. Quaternary ammonium salts are widely used in industry and medicine [5] and many antiseptic solutions contain them. However, there is a known incompatibility between HA and quaternary ammonium 
salts (QAS) due to the risk of HA precipitation in the solution [6]. Therefore, many instructions for use of HA viscosupplements recommend to avoid the use of QAS to disinfect the injection site. This is a concern in patients with allergies to iodine, as most other skin antiseptics are made from iodine derivatives. However, in the conditions of clinical practice, it has never been evidenced that QAS disinfectants exert a negative impact on the rheological properties of viscosupplements.

The aim of the present study was to demonstrate that the use of QAS for skin disinfection does not modify the rheological properties of HA solutions and, consequently, should not be contra-indicated in viscosupplémentation.

\section{Materials and Methods}

The rheological properties of 2 HA viscosuppléments combined to mannitol, both demonstrated to be effective in knee osteoarthritis, HANOX-M-XL, a cross-linked HA (16 mg/ml) (HAppyCross ${ }^{\circ}$ LABRHA SAS, Lyon, France) [7] and HANOX-M, a linear HA (15.5 mg/ml) (HAppyVisc ${ }^{\circ}$ LABRHA SAS, Lyon, France) [8], were measured in triplicates using a cone-plate rheometer (RheoWin HAAKE Viscotester iQ Air, Thermo Electron SAS) at $20{ }^{\circ} \mathrm{C}$. One milliliter of each viscosupplement was placed on the rheometer plate with the use of a $21 \mathrm{G}$ x $40 \mathrm{~mm}$ needle (BD microfine ${ }^{\circ}$ ) through a piece of pork skin previously disinfected with Biseptine (Bayer Healthcare, France), an antiseptic from the QAS family (chlorhexidine gluconate, benzalkonium chloride and benzyl alcohol) or disinfected with povidone, an iodinated derivative (Betadine dermique $10 \%$, Mylan Medical SAS, France) or not disinfected. The same procedure without the pork skin (HA directly placed on the rheometer plate) was used as control. Before starting measurements, the sample was deposited on the bottom plane of the rheometer and spread using the upper cone. Product surplus was removed with a spatula or syringe. The air gap was then adjusted, and the sample conditioned at a temperature of 20 ${ }^{\circ} \mathrm{C}$ with a precision of $0.5^{\circ} \mathrm{C}$. The dynamic viscosity $\eta$ (Pa.s) of both viscosuppléments was determined according to the shear rate $\gamma$ from 0.1 to $100 \mathrm{~s}^{-1}$. The results are given with an accuracy of $10 \%$ whereas the imperfections of the geometries and the precision of the rheometer.

\section{Results}

As expected the crosslinked viscosupplement exhibited higher viscosity than the linear one at all share rates. As shown by the flow curves (Figures $1 \& 2$ ), the steady-state viscosity as a function of the shear rate of HANOX-M-XL and HANOX-M were strictly similar regardless of the skin disinfection protocol. Table 1 shows the dynamic viscosity of HANOX-M-XL at a shear rate of $0.5 \mathrm{~s}^{-1}$ and $50 \mathrm{~s}^{-1}$. (Table 2) shows the dynamic viscosity of HANOX-M at a shear rate of $0.5 \mathrm{~s}^{-1}$ and $50 \mathrm{~s}^{-1}$. Whether the viscosupplement was crosslinked or not, the dynamic viscosity was not significantly altered by the skin disinfection protocol (all p-values>0.05).

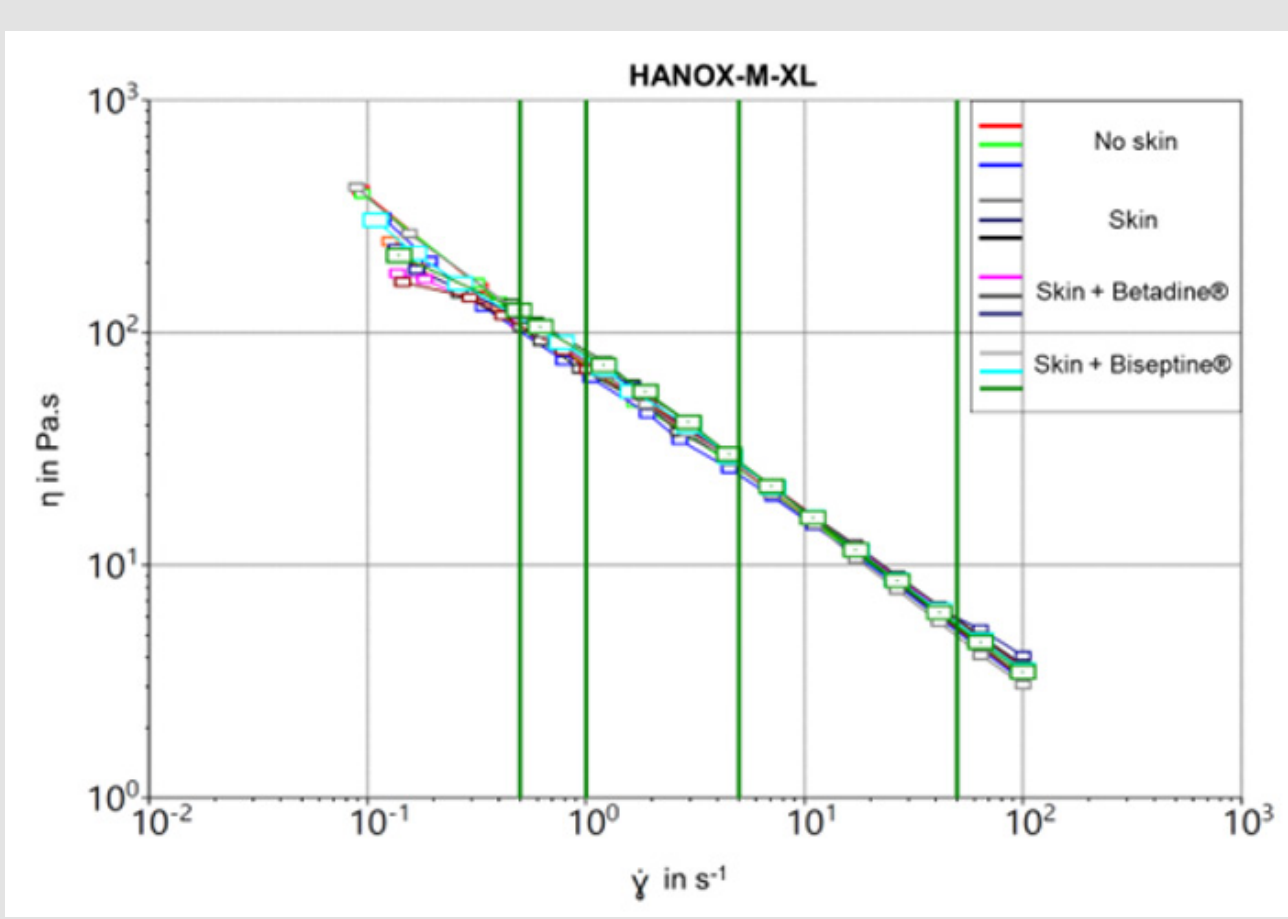

Figure 1: Steady-state viscosity of crosslinked HA,HANOX-M-XL, as a function of the shear rate for the different skin disinfection protocols. 


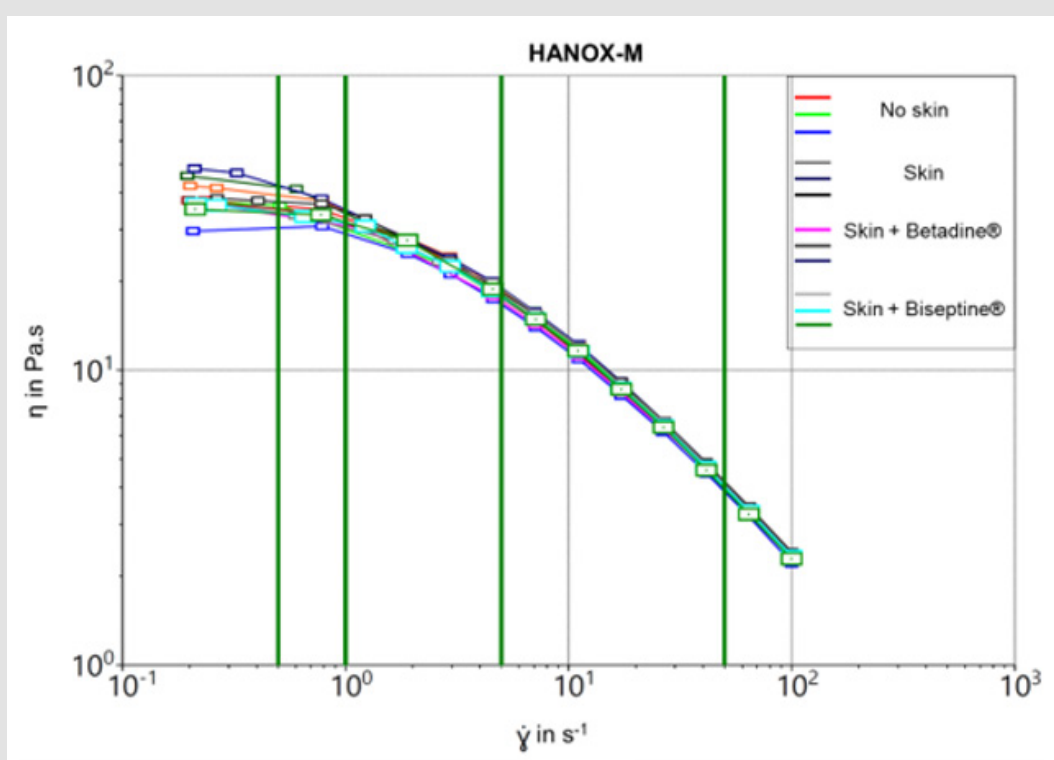

Figure 2: Steady-state viscosity of linear HA, HANOX-M, as a function of the shear rate for the different skin disinfection protocols.

Table 1: Dynamic viscosity $\eta(S D)$ of HANOX-M-XL according to the method of skin disinfection at a shear rate of $0.5 \mathrm{~s}^{-1}$ and $50 \mathrm{~s}^{-1}$.

\begin{tabular}{|c|c|c|c|c|c|}
\hline & No Skin & Skin & Skin + Biseptine ${ }^{\circledR}$ & Skin + Betadine $^{\circledR}$ & P Values \\
\hline $\begin{array}{c}\text { Mean } \eta(P a . s) \\
\gamma=0.5 \mathrm{~s}^{-1}\end{array}$ & $110.8(9.2)$ & $116.2(5.9)$ & $109.8(2.8)$ & $114.4(4.9)$ & 0.2 \\
\hline $\begin{array}{c}\text { Mean } \eta(\text { Pa.s) } \\
\gamma=50 \mathrm{~s}^{-1}\end{array}$ & $5.3(0.1)$ & $5.7(0.3)$ & $5.8(0.1)$ & $5.5(0.4)$ & 0.4 \\
\hline
\end{tabular}

Table 2: Dynamic viscosity $\eta(S D)$ of HANOX-M according to the method of skin disinfection at a shear rate of $0.5 \mathrm{~s}^{-1}$ and $50 \mathrm{~s}^{-1}$.

\begin{tabular}{|c|c|c|c|c|c|}
\hline & No Skin & Pork Skin & Pork Skin + Betadine & Pork Skin + Biseptine & P Values \\
\hline $\begin{array}{c}\text { Mean } \eta \text { (Pa.s) } \\
\gamma=0.5 \mathrm{~s}^{-1}\end{array}$ & $34.2(3.3)$ & $39.2(1.7)$ & $35.4(1.8)$ & $33.8(0.1)$ & 0.4 \\
\hline $\begin{array}{c}\text { Mean } \eta(\text { Pa.s) } \\
\gamma=50 \mathrm{~s}^{-1}\end{array}$ & $4.1(0.2)$ & $4.2(0.1)$ & $4.1(0.1)$ & $4.0(0.1)$ & 0.7 \\
\hline
\end{tabular}

\section{Discussion}

We showed that the viscous behaviour of 2 HA viscosuppléments, linear and crosslinked, did not change regarding the skin disinfection modalities. The dynamic viscosity was the same whether the viscosupplement was applied directly to the rheometer, through a not-disinfected skin, or through a skin disinfected with a QAS or an iodine derivative. Rheological examination is a sensitive and reliable tool for assessing structural changes of HA. The viscoelastic properties of linear HA solutions are strictly related to the product of molar mass and HA concentration, which also controls HA's biologic effects $[9,10]$. In crosslinked HA the elasto-viscous behaviour is mainly related to the density of the entangled network. Consequently, at a constant HA concentration, any reduction of the intrinsic viscosity reflects a decrease of its molecular weight. In our experiment, since HA concentration was constant, we can assert that the skin disinfection with Biseptine ${ }^{\circ}$ did not induce structural changes (i.e., depolymerization) of the HA macromolecule. This is obviously due to the fact that HA is never in direct contact with the QAS. Either way, even if it did, the amount of QAS would be too low, with most of the solution evaporating from the skin when the needle is inserted into the joint. In summary, skin disinfection with QAS does not modify the rheological properties of HA viscosuppléments. The use of QAS should no longer be contraindicated for skin disinfection before HA intra-articular injection. The present data allow to reassure practitioners achieving viscosupplémentation with regard to the use of QAS disinfectants in patients with iodinated derivative allergy.

\section{Disclosure of Interests}

Elise Murat is an employee of Laboratoire de rhumatologie Appliquée. Thierry Conrozier received fees from Medac, Fidia, Labrha and Sanofi for consultant and speaker services. 


\section{References}

1. Maheu E, Bannuru RR, Herrero Beaumont G, Allali F, Bard H, et al. (2019) Why we should definitely include intra-articular hyaluronic acid as a therapeutic option in the management of knee osteoarthritis: Results of an extensive critical literature review. Semin Arthritis Rheum 48(4): 563-572.

2. Legré Boyer V (2015) Viscosupplementation: techniques, indications, results. Orthop Traumatol Surg Res 101(1): S101-108.

3. Adams ME, Lussier AJ, Peyron JG (2000) A risk-benefit assessment of injections of hyaluronan and its derivatives in the treatment of osteoarthritis of the knee. Drug Saf 23(2): 115-130.

4. Holland C, Jaeger L, Smentkowski U, Weber B, Otto C (2012) Septic and aseptic complications of corticosteroid injections: an assessment of 278 cases reviewed by expert commissions and mediation boards from 2005 to 2009. Dtsch Arztebl Int 109(24): 425-430.

5. Oblak E, Piecuch A, Rewak Soroczynska J, Paluch E (2019) Activity of gemini quaternary ammonium salts against microorganisms. Applied Microbiology and Biotechnology 103(2): 625-632.

ISSN: 2574-1241

DOI: 10.26717/BJSTR.2021.35.005728

Thierry Conrozier. Biomed J Sci \& Tech Res

(C) (P) This work is licensed under Creative

Submission Link: https://biomedres.us/submit-manuscript.php
6. W Jeanloz R, Forchielli E (1950) Studies on hyaluronic acid and related substances: I. Preparation of hyaluronic acid and derivatives from human umbilical cord. J Biol Chem 186(2): 495-511.

7. Henrotin Y, Berenbaum F, Chevalier X, Marty M, Richette P, et al. (2017) Reduction of the Serum Levels of a Specific Biomarker of Cartilage Degradation (Coll2-1) by Hyaluronic Acid (KARTILAGE ${ }^{\circ}$ CROSS) Compared to Placebo in Painful Knee Osteoarthritis Patients: the EPIKART Study, a Pilot Prospective Comparative Randomized Double Blind Trial. BMC Musculoskelet Disord 18(1): 222.

8. Conrozier T, Eymard F, Afif N, Balblanc JC, Legré Boyer V, et al. (2016) Study Group (2016) Safety and efficacy of intra-articular injections of a combination of hyaluronic acid and mannitol (HAnOX-M) in patients with symptomatic knee osteoarthritis: Results of a double-blind, controlled, multicenter, randomized trial. Knee 23(5): 842-848.

9. Berriaud N, Milas M, Rinaudo M (1994) Rheological study on mixtures of different molecular weight hyaluronates. Int J Biol Macromol 16(3): 137-142.

10. Mathieu P, Conrozier T, Vignon E, Rozand Y, Rinaudo M (2009) Rheologic behavior of osteoarthritic synovial fluid after addition of hyaluronic acid: a pilot study. Clin Orthop Relat Res 467(11): 3002-3009.

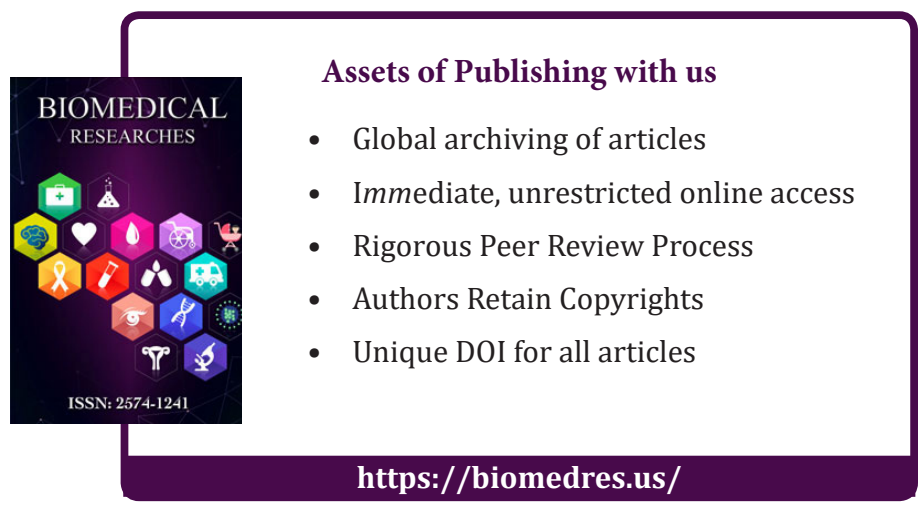

